\title{
Delayed Inhibition of c-Jun N-Terminal Kinase Worsens Outcomes after Focal Cerebral Ischemia
}

\author{
Yoshihiro Murata, ${ }^{1}$ Norio Fujiwara, ${ }^{1}$ Ji Hae Seo, ${ }^{1}$ Feng Yan, ${ }^{2}$ Xiangrong Liu, ${ }^{2}$ Yasukazu Terasaki, ${ }^{1}$ Yumin Luo, ${ }^{2}$ \\ Ken Arai, ${ }^{1}$ Xunming Ji, ${ }^{2}$ and Eng H. Lo ${ }^{1}$ \\ ${ }^{1}$ Neuroprotection Research Laboratory, Departments of Radiology and Neurology, Massachusetts General Hospital, and Program in Neuroscience, Harvard \\ Medical School, Boston, Massachusetts 02129 and ${ }^{2}$ Cerebrovascular Research Institute and Departments of Neurology and Neurosurgery, Xuanwu \\ Hospital, Capital Medical University, Beijing 100053, China
}

The stress-activated protein kinase c-Jun N-terminal kinase (JNK) is a central regulator in neuronal death cascades. In animal models of cerebral ischemia, acute inhibition of JNK reduces infarction and improves outcomes. Recently however, emerging data suggest that many neuronal death mediators may have biphasic properties-deleterious in the acute stage but potentially beneficial in the delayed stage. Here, we hypothesized that JNK may also have biphasic actions, so some caution may be required in the development of JNK inhibitors for stroke. Sprague Dawley rats underwent 90 min transient occlusions of the middle cerebral artery. Acute treatment (10 min poststroke) with the JNK inhibitor SP600125 reduced infarction volumes. In contrast, delayed treatment (7 d poststroke) worsened infarction volumes and neurological outcomes. Immunostaining of peri-infarct cortex showed that JNK inhibition suppressed surrogate markers of neurovascular remodeling, including matrix metalloproteinase- 9 in GFAP-positive astrocytes and microvascular density. Consistent with these in vivo data, SP600125 significantly suppressed in vitro angiogenesis in rat brain endothelial cultures. Our data provide initial proof-of-concept that the neuronal death target JNK may also participate in endogenous processes of neurovascular remodeling and recovery after cerebral ischemia.

\section{Introduction}

An emerging idea in stroke suggests that many mediators and mechanisms triggered by cerebral injury may play biphasic roles, i.e., contribute to tissue damage during the acute phase but mediate repair during delayed recovery (Lo, 2008; Hurtado et al., 2011). For example, overactivation of NMDA receptors is excitotoxic during acute stroke or traumatic brain injury (Besancon et al., 2008). But conversely, NMDA signaling may underlie neuronal plasticity during stroke recovery (McKinney, 2010). Similarly, matrix metalloproteinases (MMPs) cause neurovascular damage during early times after stroke (Candelario-Jalil et al., 2009). But MMPs are essential for neurovascular remodeling during later phases of endogenous recovery (Zhao et al., 2006).

It is increasingly recognized that this biphasic nature of molecular mediators may be a basic principle that broadly applies to all of stroke pathophysiology. Here, we ask whether the same phenomenon may also be manifested in neuronal death pathways. The stress-activated protein kinase c-Jun N-terminal kinase

\footnotetext{
Received Jan. 16, 2012; revised March 8, 2012; accepted March 25, 2012.

Author contributions: Y.M., N.F., Y.L., K.A., X.J., and E.H.L. designed research;Y.M., N.F., J.H.S., F.Y., X.L., and Y.T. performed research; Y.M., N.F., J.H.S., F.Y., X.L., Y.L., K.A., and X.J. analyzed data; Y.M., N.F., Y.L., K.A., X.J., and E.H.L. wrote the paper.

This work was supported in part by NIH Grants R37-NS37074 and P01-NS55104, Grant 2011CB707804 from the National Basic Research Program of China 973 Program, and Grant 7111003 from the Beijing Natural Science Foundation.

The authors declare no competing financial interests.

Correspondence should be addressed to Eng H. Lo, MGH East 149-2401, Charlestown, MA 02129. E-mail: Lo@helix.mgh.harvard.edu.

DOI:10.1523/JNEUROSCI.0219-12.2012

Copyright $\odot 2012$ the authors $\quad 0270-6474 / 12 / 328112-04 \$ 15.00 / 0$
}

(JNK) is a critical regulator of neuronal cell death (Vosler and Chen, 2009). Blockade of JNK has been shown to reduce infarct size in models of cerebral ischemia (Borsello et al., 2003; Gao et al., 2005). But is it possible that JNK may also somehow contribute to stroke recovery?

\section{Materials and Methods}

Rat focal cerebral ischemia model. Following institutionally approved protocols, male Sprague Dawley rats were subjected to transient focal cerebral ischemia under isofluorane anesthesia. Using a standard approach (Murata et al., 2008), intraluminal filaments were used to occlude the middle cerebral artery for $90 \mathrm{~min}$. Laser-doppler flowmetry was used to confirm successful occlusions ( $<20 \%$ baseline) and reperfusion. Physiologic parameters were confirmed to lie in normal ranges via blood sampled from femoral catheters $(95-110 \mathrm{mmHg}$ blood pressure, $7.32-7.36$ $\mathrm{pH}, 110-140 \mathrm{mmHg}_{2}, 40-45 \mathrm{mmHg}_{\mathrm{pCO}}$ ). Vehicle (10 $\mu \mathrm{l}$ of $1 \%$ DMSO in PBS) or the JNK inhibitor SP600125 (30 $\mu \mathrm{g}$ in $10 \mu \mathrm{l}$ of $1 \%$ DMSO in PBS) was injected into the lateral ventricles. This dosing of SP600125 was selected based on previously published studies that used this compound to target JNK in rodent models in vivo (Guan et al., 2005). Intraventricular injections ensured that drug reached the brain in our animal models. It is important to remember that these routes may not be relevant to human patients where intravenous drug delivery is used. To compare effects of JNK inhibition on acute injury versus delayed repair, two different treatment protocols were used. For acute treatments, drugs were administered $10 \mathrm{~min}$ after ischemic onset, and at $24 \mathrm{~h}$, rats were assessed with a five-point neurological scale (Murata et al., 2008) before brains were measured for infarction with standard tetrazolium staining. For delayed treatments, drugs were administered on day 7 postischemia, and on day 10 , rats were assessed with a five-point neurological scale and brains were removed for hematoxylin-eosin staining. Treatment groups 
A

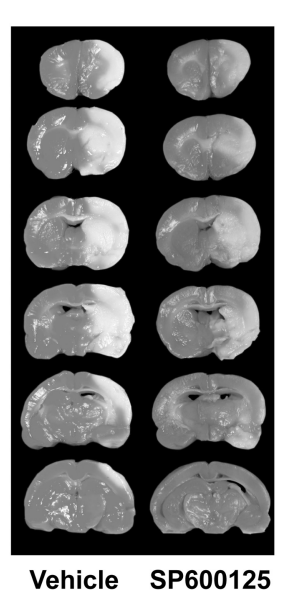

B

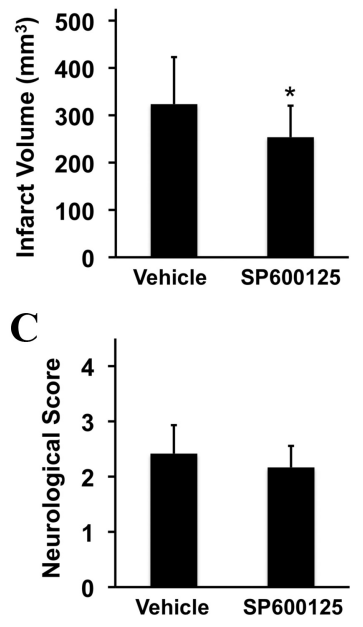

Figure 1. Effects of early ( $10 \mathrm{~min}$ poststroke onset) JNK inhibition on acute $24 \mathrm{~h}$ outcomes after focal cerebral ischemia in rats. $\boldsymbol{A}$, Representative tetrazolium-stained axial sections that define focal ischemic brain injury. $\boldsymbol{B}$, Infarct volumes at $24 \mathrm{~h}\left(\mathrm{~mm}^{3}\right.$; mean $\left.+\mathrm{SD}\right) ;{ }^{*} p<0.05$ comparing vehicle controls versus SP600125-treated rats ( $n=12$ per group). $C$, Neurological scoring (mean + SD).

were assigned and assessments were performed in a randomized and blinded manner.

Immunostaining. At $10 \mathrm{~d}$ poststroke, brains were removed and immunostaining was used to assess levels of GFAP-positive astrocytic density (1:200; BD Biosciences), RECA-1-positive vascular density (1:200; Serotec), and matrix metalloproteinase-9 (1:200; AbCam). Immunostaining signals were quantified in peri-infarct cortical regions of interest (ROIs) using standard densitometry methods in NIH ImageJ. Three peri-infarct ROIs surrounding the infarction boundary for each immunostained section were selected randomly. Six sections were assessed per brain for each animal from each treatment group.

In vitro angiogenesis. To measure the effects of JNK in angiogenesis, the standard Matrigel assay was used to assess the formation of capillarylike structures, i.e., tube formation, by rat brain endothelial cells (RBE4). Standard 24-well plates were coated with $150 \mu \mathrm{l}$ of cold Matrigel (BD Biosciences) and allowed to solidify at $37^{\circ} \mathrm{C}$ for $30 \mathrm{~min}$. Cells $\left(2 \times 10^{4}\right.$ cells $/ \mathrm{cm}^{2}$ ) were seeded in the plates and incubated at $37^{\circ} \mathrm{C}$ for $18 \mathrm{~h}$ with or without growth factor supplements. This growth factor stimulation of endothelial Matrigel tube formation was performed using standard reagents, i.e., the EGM2 medium containing the EGM2 MV supplemental kit (catalog no. CC-4177; Lonza), which comprises a mix of FBS, VEGF, EGF, IGF-1, and FGF-2. SP600125 was added at 1 and $3 \mu \mathrm{l} / \mathrm{ml}$ concentrations. The concentration of SP600125 stock solution was $10 \mathrm{~mm}$ in DMSO. To prepare final concentrations, stock solutions were diluted in culture media reaching final DMSO concentrations of $0.1 \%$, which did not affect cellular viability. The same $0.1 \%$ DMSO solutions were always used as vehicle controls. Tubes were counted in four random fields from each well and expressed as a percentage of the tubes in untreated control wells.

Statistics. Results were analyzed with $t$ tests for parametric data and Mann-Whitney for nonparametric data. Differences at $p<0.05$ were considered significant.

\section{Results}

In the acute treatment study, vehicle-treated controls were compared with rats treated with the JNK inhibitor SP600125 at 10 min poststroke onset. At $24 \mathrm{~h}$, well defined infarctions were present in the middle cerebral artery territory encompassing striatum and cortex (Fig. 1A). As expected, JNK inhibition was neuroprotective and infarct volumes were significantly reduced (Fig. $1 B$ ). All rats showed clear neurological deficits at $24 \mathrm{~h}$, but there was

A

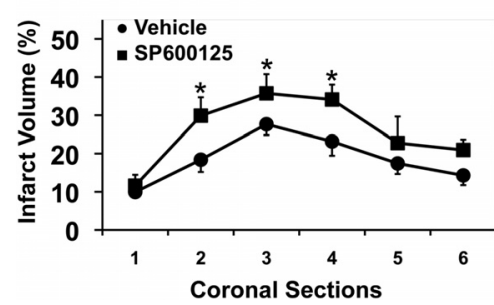

B

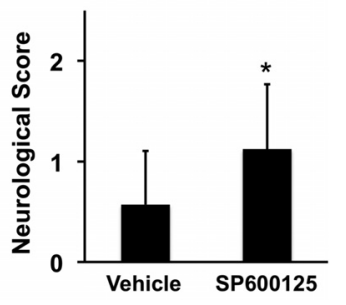

Figure 2. Effects of delayed (7 d poststroke onset) JNK inhibition on long-term $10 \mathrm{~d}$ outcomes after focal cerebral ischemia in rats. $\boldsymbol{A}$, Infarct areas measured over six axial sections at $10 \mathrm{~d}$ (percent ipsilateral hemisphere; mean + SD); ${ }^{*} p<0.05$ comparing vehicle controls versus SP600125-treated rats ( $n=8$ per group). $\boldsymbol{B}$, Neurological scoring (mean + SD); ${ }^{*} p<$ 0.05 comparing vehicle controls versus SP600125-treated rats ( $n=8$ per group).

no detectable difference between rats treated with the JNK inhibitor versus vehicle controls (Fig. 1C).

In the delayed treatment study, rats were treated with vehicle or the JNK inhibitor at $7 \mathrm{~d}$ poststroke onset, and then outcomes were assessed at $10 \mathrm{~d}$. When brains were removed for histological analysis, infarction volumes were significantly enlarged by delayed JNK inhibition, especially in the central axial sections of the middle cerebral artery territory (Fig. $2 A$ ). All rats showed persistent neurological deficits. But rats treated with the JNK inhibitor appeared to be more impaired compared with vehicle-treated rats (Fig. $2 B$ ).

To understand why JNK inhibition worsened outcomes, brains were subjected to immunostaining. As expected, the periinfarct cortex at the $10 \mathrm{~d}$ time point showed evidence of new microvessel formation, comprising Ki67 signals colocalized with CD31-positive structures (Fig. 3A). To quantify these responses, several surrogate markers of neurovascular remodeling were examined, including increased density of RECA-1-positive microvessels (Fig. 3B) and GFAP-positive astrocytes with augmented MMP-9 levels (Fig. 3C). Rats treated with the JNK inhibitor appeared to have somewhat decreased immunostaining signals. Quantitative densitometry showed that peri-infarct microvessel density (Fig. 3D) and MMP-9 signals (Fig. 3E) were significantly reduced by JNK inhibition.

Since these in vivo findings suggested a deleterious effect of JNK inhibition on neurovascular recovery poststroke, we next asked whether JNK was required for angiogenesis in cerebral endothelium. Rat brain endothelial cells (RBE4) were seeded and the Matrigel assay was used as an in vitro angiogenesis assay. Treatment with growth factor supplements promoted tube formation (Fig. $4 A$ ). Inhibition of JNK with 1-3 $\mu \mathrm{M}$ SP600125 clearly suppressed tube formation (Fig. $4 B$ ) without any evidence of cytotoxicity (Fig. 4C). These antiangiogenic effects of SP600125 were confirmed with Western blots. Stimulated Matrigel tube formation involved elevations in phospho-JNK levels, and blockade with SP600125 reduced phospho-JNK levels (Fig. 4D).

\section{Discussion}

In a wide range of molecular, cellular, and whole-animal model systems, JNK has emerged as a central regulator and integrator of multiple cell death pathways (Vosler and Chen, 2009). Blockade of JNK has been shown to be efficacious in many animal models of cerebral ischemia (Borsello et al., 2003; Gao et al., 2005). Based on these cellular and preclinical data, there has been some movement toward the pursuit of JNK inhibitors for stroke. Our proof-of-concept study here suggests that some caution 

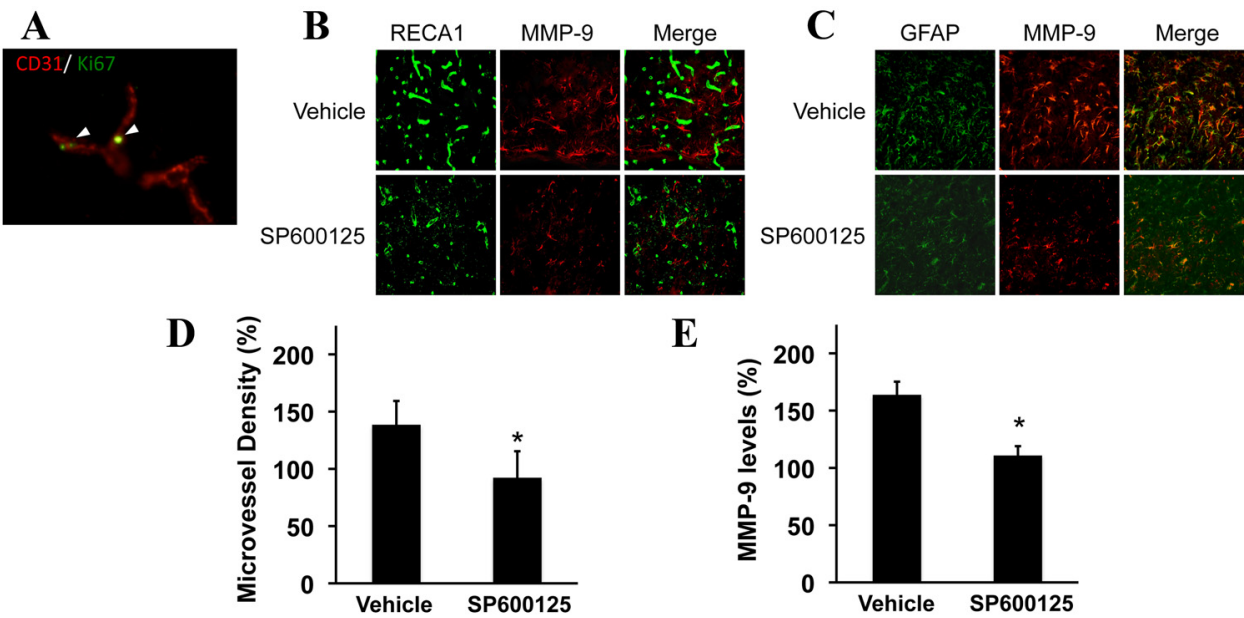

Figure 3. Immunostaining of neurovascular remodeling in peri-infarct cortex. A, Ki67 colocalization with CD31-positive endothelial cells suggested the presence of new microvessel formation during the delayed recovery phase of the peri-infacrt cortex. $\boldsymbol{B}$, RECA-positive microvessels were reduced by JNK inhibition. $\boldsymbol{C}$, MMP-9 levels in GFAP-positive astrocytes were reduced by JNK inhibition. D, Quantitative densitometry of RECA immunostaining. $E$, Quantitative densitometry of MMP-9 immunostaining. ${ }^{*} p<0.05$ comparing vehicle controls versus SP600125-treated rats ( $n=8$ per group).

A
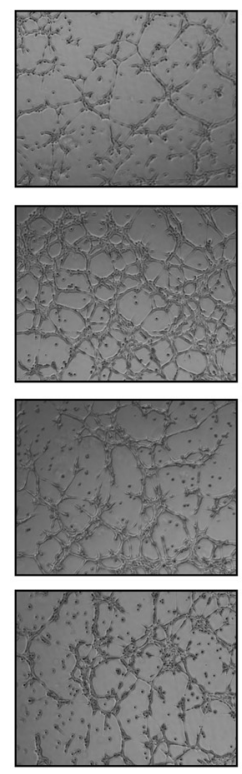

Control

Stimulation

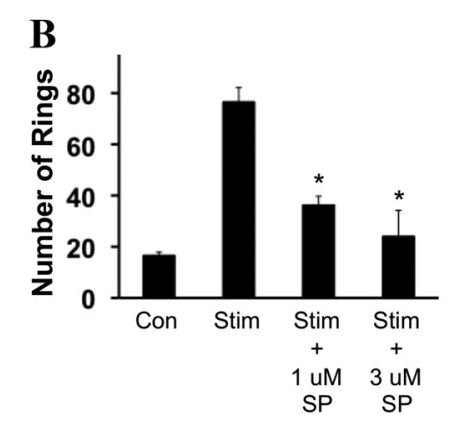

C
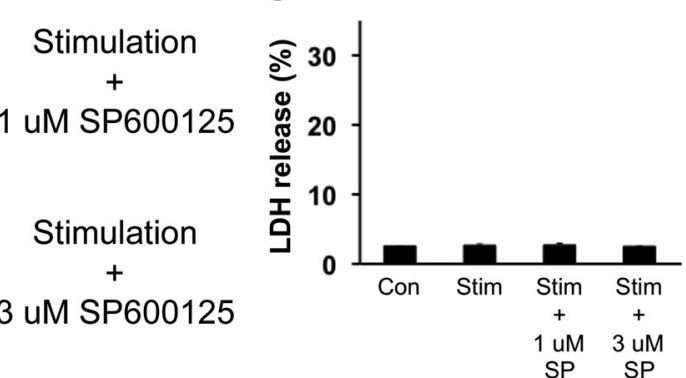

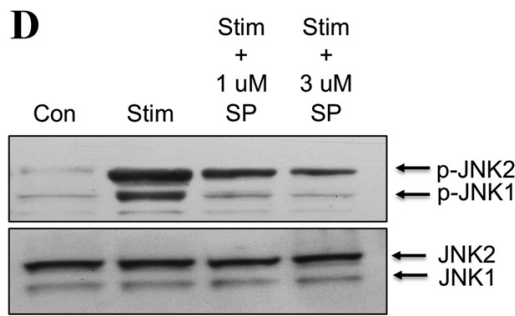

SP SP

Figure 4. Effects of JNK inhibition on in vitro angiogenesis. A, Representative Matrigel tube formation images in RBE4 rat brain endothelial cells. $B$, Quantified tube formation rates. ${ }^{*} p<0.05$ comparing vehicle controls versus SP600125-treated rats ( $n=3$ independent experiments in triplicate). C, SP600125 was not cytotoxic according to a standard LDH release assay. D, Western blots showed increased phospho-JNK during Matrigel tube formation. Reduction of tube formation by SP600125 was accompanied by suppression of phospho-JNK. Con, Control; Stim, stimulation; SP, SP600125; p, phospho.

may be warranted. As expected, acute JNK inhibition was neuroprotective. But blocking JNK at later stages of stroke evolution seemed to interfere with neurovascular remodeling and functional recovery.

JNK is an evolutionarily conserved signaling mechanism that performs multiple functions across the phylogenetic spectrum. In many systems, ranging from insects to mammals, JNK plays diverse roles in organ development and tissue repair. In Drosophila, JNK homologs contribute to cell proliferation and wound healing (Bosch et al., 2005). In Caenorhabditis elegans, JNK homologs participate in normal development as well as in stress response after injury (Mizuno et al., 2004). In mammals, JNK is known to play a role in neural development and dendritic sprouting
(Waetzig et al., 2006). Functionally, JNK is an important part of learning and memory systems (Sherrin et al., 2011). And there is at least one study suggesting that JNK may mediate angiogenesis in human umbilical vein endothelial cells (Wu et al., 2006). Our present findings might be consistent with this broader literature. After stroke, JNK's proangiogenic actions may augment neurovascular remodeling and brain repair. If one seeks to block JNK for neuroprotection, care has to be taken so as to not interfere with JNK's beneficial actions in neurovascular recovery.

If JNK signaling plays biphasic roles after stroke, then an important follow-up question is how the cellular profiles of these mechanisms evolve over time. During the acute phase, JNK is rapidly activated in neurons, thus leading to neuronal cell death 
(Okuno et al., 2004). In our model, we also observe phospho-JNK in acute ischemic brain homogenates. But how does JNK continue to operate as the brain transitions from injury into repair? Our initial findings suggest that within peri-infarct cortex, phopho-JNK can still be detected during delayed stages of stroke recovery, in part associated with microvessel-like structures. But how these signals mediate crosstalk between multiple cell types of the entire neurovascular unit remains to be carefully dissected in future studies. Furthermore, the timescale of infarct maturation and peri-infarct recovery will be different between human stroke patients and animal models. In this study, we used a standard intraluminal filament model of focal ischemia. In this model, infarction matures very quickly. It would be surprising if infarction continued to slowly evolve after $7-10 \mathrm{~d}$. Instead, what might be happening is that in the 7-10 d peri-infarct zone, endogenous repair mechanisms are dependent on JNK to help maintain the remodeling penumbra. Interfering with JNK-mediated endogenous mechanisms of neurovascular plasticity then makes the tissue devolve into further damage.

The idea of biphasic effects of JNK may have implications beyond stroke per se. JNK has also been proposed as a potential neuroprotective target in Alzheimer's disease and Parkinson's disease (Repici and Borsello, 2006). Neurovascular homeostasis is critically involved in a wide range of CNS disorders (Iadecola, 2004; Zlokovic, 2008). A dynamic balance between neurovascular injury and repair may not just underlie stroke and acute brain injury (Lo, 2008), but may also play a central role in the slower pathophysiology of neurodegeneration (Lo, 2010). Hence, the need for caution in targeting JNK may broadly extend to all CNS disorders.

Together, our data suggest that delayed inhibition of JNK with SP600125 may interfere with endogenous neurovascular remodeling, increase infarction, and worsen outcomes after stroke. Nevertheless, several caveats must be kept in mind. First, in this initial study, we only assessed a single time point poststroke and a single dose of JNK inhibition. Further experiments are needed to define the temporal course of precisely when the transition occurs between beneficial versus detrimental effects of JNK blockade, and whether different doses of SP600125 yield different outcomes remains to be determined. Second, we only looked at MMP-9 as a surrogate marker of neurovascular repair. Clearly, microvessel remodeling will involve coordinated activation of a wide range of other mediators, including trophic factors. How JNK broadly affects trophic factor profiles in the poststroke context remains unknown. Third, MAP kinase signaling pathways do not operate in isolation. How our hypothesized JNK mechanism interacts with other cascades such as ERK, p38, or Stat requires careful study in the future. SP600125 is relatively specific for JNK. Whether off-target effects occur in these other related pathways should be carefully assessed. Finally, the question of how functional recovery is correlated with morphological tissue remodeling remains unclear. In this proof-of-concept study, our endpoints were limited to gross measurements of infarct volume and relatively crude neurological scoring scales. Other, more sophisticated measurements of stroke recovery (perhaps including fine sensorimotor tasks such as pellet retrieval or cognitive/memory maze tests) will have to be applied to unequivocally dissect how JNK signaling contributes to functional repair after stroke.

Optimal development of stroke therapies may require a more careful characterization of the mechanisms by which the brain transitions from initial injury into delayed repair. In this proofof-concept study, we showed that this principle might also apply to the neuronal death mediator JNK. JNK may still be a valid target in stroke, but some caution may be required in terms of defining the dosing and timing of therapies to maximize benefit without interfering with endogenous mechanisms of recovery.

\section{References}

Besancon E, Guo S, Lok J, Tymianski M, Lo EH (2008) Beyond NMDA and AMPA glutamate receptors: emerging mechanisms for ionic imbalance and cell death in stroke. Trends Pharmacol Sci 29:268-275.

Borsello T, Clarke PG, Hirt L, Vercelli A, Repici M, Schorderet DF, Bogousslavsky J, Bonny C (2003) A peptide inhibitor of c-Jun N-terminal kinase protects against excitotoxicity and cerebral ischemia. Nat Med 9:1180-1186.

Bosch M, Serras F, Martín-Blanco E, Baguñà J (2005) JNK signaling pathway required for wound healing in regenerating Drosophila wing imaginal discs. Dev Biol 280:73-86.

Candelario-Jalil E, Yang Y, Rosenberg GA (2009) Diverse roles of matrix metalloproteinases and tissue inhibitors of metalloproteinases in neuroinflammation and cerebral ischemia. Neuroscience 158:983-994.

Gao Y, Signore AP, Yin W, Cao G, Yin XM, Sun F, Luo Y, Graham SH, Chen J (2005) Neuroprotection against focal ischemic brain injury by inhibition of c-Jun N-terminal kinase and attenuation of the mitochondrial apoptosis-signaling pathway. J Cereb Blood Flow Metab 25:694-712.

Guan QH, Pei DS, Zhang QG, Hao ZB, Xu TL, Zhang GY (2005) The neuroprotective action of SP600125, a new inhibitor of JNK, on transient brain ischemia-reperfusion-induced neuronal death in rat hippocampal CA1 via nuclear and non-nuclear pathways. Brain Res 1035:51-59.

Hurtado O, Lizasoain I, Moro MÁ (2011) Neuroprotection and recovery. Stroke 42:S33-35.

Iadecola C (2004) Neurovascular regulation in the normal brain and in Alzheimer's disease. Nat Rev Neurosci 5:347-360.

Lo EH (2008) A new penumbra: transitioning from injury into repair after stroke. Nat Med 14:497-500.

Lo EH (2010) Degeneration and repair in central nervous system disease. Nat Med 16:1205-1209.

McKinney RA (2010) Excitatory amino acid involvement in dendritic spine formation, maintenance and remodeling. J Physiol 588:107-116.

Mizuno T, Hisamoto N, Terada T, Kondo T, Adachi M, Nishida E, Kim DH, Ausubel FM, Matsumoto K (2004) The Caenorhabditis elegans MAPK phosphatase VHP-1 mediates a novel JNK-like signaling pathway in stress response. EMBO J 23:2226-2234.

Murata Y, Rosell A, Scannevin RH, Rhodes KJ, Wang X, Lo EH (2008) Extension of the thrombolytic time window with minocycline in experimental stroke. Stroke 39:3372-3377.

Okuno S, Saito A, Hayashi T, Chan PH (2004) The c-Jun-N-terminal protein kinase signaling pathway mediates Bax activation and subsequent neuronal apoptosis through interaction with Bon after transient focal cerebral ischemia. J Neurosci 24:7879-7887.

Repici M, Borsello T (2006) JNK pathway as therapeutic target to prevent degeneration in the central nervous system. Adv Exp Med Biol 588:145-155.

Sherrin T, Blank T, Todorovic C (2011) c-Jun N-terminal kinases in memory and synaptic plasticity. Rev Neurosci 22:403-410.

Vosler PS, Chen J (2009) Potential molecular targets for translational stroke research. Stroke 40:S119-120.

Waetzig V, Zhao Y, Herdegen T (2006) The bright side of JNKsmultitalented mediators in neuronal sprouting, brain development and nerve fiber regeneration. Prog Neurobiol 80:84-97.

Wu G, Luo J, Rana JS, Laham R, Sellke FW, Li J (2006) Involvement of COX-2 in VEGF-induced angiogenesis via P38 and JNK pathways in vascular endothelial cells. Cardiovasc Res 69:512-519.

Zhao BQ, Wang S, Kim HY, Storrie H, Rosen BR, Mooney DJ, Wang X, Lo EH (2006) Role of matrix metalloproteinases in delayed cortical responses after stroke. Nat Med 12:441-445.

Zlokovic BV (2008) The blood-brain barrier in health and chronic neurodegenerative disorders. Neuron 57:178-201. 